Niaz Muhammad Khan, Hayat Ullah¹, Abdur Raziq', Adnan Ali Khan², Muhammad Waseem Khan ${ }^{3}$

${ }^{1}$ Department of Bioinformatics \& Biotechnology, Faculty of Life Science, Government College University Faisalabad, Pakistan

${ }^{2}$ Department of Genetics, Hazara University Mansehra, Pakistan

${ }^{3}$ Department of Biotechnology, Faculty of Life Sciences and Informatics, Balochistan University of Information Technology Engineering and Management Sciences, Quetta, Pakistan

\title{
Molecular genetic analysis of leucine tRNA in relevance to type 2 diabetes mellitus
}

\section{ABSTRACT}

Background. Several point mutations in the mitochondrial DNA cause maternally inherited metabolic disorders. The most common type of mutation A3243G in the gene of transfer RNA leucine (tRNA ${ }^{\text {Leu(UUR) }}$ ) is thought to be responsible for the prevalence of type 2 diabetes mellitus. This study was designed to analyze the tRNA ${ }^{\text {Leu(UUR) }}$ gene of mtDNA of the diabetic individuals with familial history of diabetes to identify the point mutations A3243G.

Material and methods. Saliva samples were preferred as a source of DNA to minimize the risk of infection. DNA was successfully extracted from their saliva. Samples of high-quality DNA was amplified with PCR and sequenced in Macrogen Inc. Korea.

Results. The m.3243A>G mutation in mitochondrial $t R N A^{\text {Leu(UUR) }}$ gene was not observed.

Conclusion. The result shows that the m.3243A>G mutation in mitochondrial tRNA ${ }^{\text {Leu(UUR) }}$ gene is not frequent cause of type 2 and some other factors may be possible i.e. genetic, behavioral or environmental. It is recommended that the sample size for diabetic individuals need to be increased for a future study

Address for correspondence:

Muhammad Waseem Khan

Department of Biotechnology

Faculty of Life Sciences and Informatics

Balochistan University of Information Technology Engineering

and Management Sciences

87300, Quetta, Pakistan

Phone: +92-333-7896575

e-mail: muhammad.waseem@buitms.edu.pk

Clinical Diabetology 2020, 3, 167-173

DOI: $10.5603 /$ DK.2020.0018

Received: 01.10.2019

Accepted: 04.05.2020 and screened for the mitochondrial as well as other mutations of nuclear origin. (Clin Diabetol 2020; 9; 3: 167-173)

Key words: diabetes mellitus, leucine tRNA, mitochondrial DNA, point mutation

\section{Introduction}

Diabetes mellitus (DM) is a common disease affecting many individuals worldwide. DM encompasses a range of metabolic failures, characterized by hyperglycemia developed from impairment of beta cells of pancreas and mutations in genomic DNA. Genetic impairment is involved in both peripheral insulin sensitivity and glucose linked insulin secretions. Mitochondria has important role in glucose insulin secretion, mutations in mitochondrial DNA (mtDNA) can cause insulin secretion impairment. In mitochondria, oxidative phosphorylation variation in the ration of intracellular adenosine triphosphate/adenosine diphosphate (ATP/ADP) may trigger the exocytosis of insulin [1].

Mitochondrial DNA follows a maternal pattern of inheritance [2]. More than 220 mutations have been related with syndromes in the 22 genes of human tRNA and 40 in human tRNA ${ }^{\text {Leu(UUR) }}$ gene. Epidemiological studies indicate prevalence of approximately 1 in 5,000 mtDNA mutations in adults, making mtDNA the most common carriers of genetic disorders [3]. Mutations in mitochondrial tRNA genes are linked with multiple human diseases, including heart failure, neuromuscular disorders, diabetes, visual and hearing loss. Particularly, mutation at position A3243G of tRNA ${ }^{\text {Leu(UUR) }}$ gene causing MELAS Syndrome (mitochondrial encephalomyopathy, lactic acidosis, and stroke-like episodes) is 
responsible for about $2 \%$ of cases of type 2 diabetes [4]. Point mutation A3243G in the tRNA ${ }^{\text {Leu(UUR) }}$ gene is one of the most common mtDNA defect, firstly diagnosed in children with MELAS Syndrome. Some families also express diabetes and deafness known as MIDD (maternally inherited diabetes and deafness) [5, 6]. In general population, MIDD is responsible for 0.5 to $1.5 \%$ of all diabetes cases. MIDD pathophysiological mechanism are complex and may involve impairment of glucose toxicity, insulin secretion and resistance. In mitochondrial diabetes, insulin impairment response to glucose challenge is in an early stage and indicates major factor in the pathogenesis of hyperglycemia. However, insulin response to glucagon and arginine rather than glucose is not clear $[7,8]$.

The most notorious mutation is A3243G in tRNA $^{\text {Leu(UUR) }}$ gene that causes impairment in the arrangement of functional proteins of Electron Transport Chain (ETC). This type of mutation is non-adoptive and brings impairment in the production of insulin [9]. A3243G point mutation becomes pathogenic when its concentration reaches $10-30 \%$.Most commonly, considered as the frequent cause of insulin independent diabetes mellitus [10]. Mutation in tRNA ${ }^{\text {Leu(UUR) }}$ gene at position A3243G cause defects in function of tRNA to properly assemble proteins in respiratory chain complexes due to which oxidative phosphorylation will not properly produce enough ATP to activate ATP-sensitive potassium channels in the $\beta$-cells to secrete insulin in response to high blood sugar level which will eventually cause type $2 \mathrm{DM}$. The Adenine to Guanine at nucleotide position A3243G in the tRNA gene play a major role in the methylation amino-acylation codon recognition and tertiary union of the molecules assembly. The mutation responsible for this maternally inherited diabetes mellitus is varying in different ethnic groups [11, 12].

The population of tribal areas of Pakistan is less explored and little literature is available on this specific ethnic group. Therefore, this study population has been assessed for mitochondrial DNA mutation which is more frequently found in population affected with type 2 diabetes mellitus. The aim of this study was to determine mitochondrial tRNA ${ }^{\text {Leu(UUR) }}$ gene mutation in MIDD in the population of tribal areas of Pakistan as this might help to determine the molecular mechanism of the type 2 DM. Such type of finding will pave the way for better treatment, genetic counselling and prenatal diagnosis of maternally inherited diabetes.

\section{Materials and methods}

The approval of this study was obtained from the Hazara University Institutional Review Board and written informed consent was obtained from all the study individuals. A detailed medical history of the study individuals was recorded and detailed pedigree (Figure 1, Table 1) was drawn at the time of visit to the affected families. Saliva samples $(5 \mathrm{ml})$ were obtained from fifteen diabetic individuals and DNA was extracted successfully. Only five $(\mathrm{N}=5)$ samples had optimum quantity of DNA required for sequencing. The collected amount of saliva $5 \mathrm{ml}$ was important to get high amount of buccal epithelial cells for high concentration of the genomic DNA. The samples were stored at $-20^{\circ} \mathrm{C}$ soon after collection to recede the risk of contamination.

\section{DNA extraction and PCR amplification}

Genomic DNA was extracted from buccal epithelial cells using a modified protocol [13]. The extracted DNA was quantified using NanoDrop measurement at ratio of absorbance at 260/280 nm as shown in Table 2 . After quantification whole genomic mitochondrial DNA was amplified using standard kit method (Qiagen Repli-G mitochondrial DNA kit). The fragments encompassing np 3243 of mitochondrial DNA were amplified with PCR using AmpliTaq DNA polymerase. The sets of reverse and forward primer were used. The nucleotide sequence of forward primer was F-5'-CAAATTCCTCCCTGTACGAAAGG-3' and the reverse primer was R-5'-AATGAGGAGTAGGAGGTTGGCC-3'. PCR was carried out in a total volume of $25 \mu \mathrm{l}$ master mix containing $50 \mathrm{ng}$ of extracted DNA, $2.0 \mu \mathrm{l}$ each dNTP, $11.5 \mu \mathrm{lddH} 2 \mathrm{O}$, $2.5 \mu \mathrm{l} \mathrm{MgCl} 2$, and $0.5 \mu \mathrm{l}$ of Taq polymerase. The DNA was initially denatured at $94^{\circ} \mathrm{C}$ for $3 \mathrm{~min}$ and subjected to $35 \mathrm{PCR}$ cycles of $94^{\circ} \mathrm{C}$ for $45 \mathrm{sec}, 59^{\circ} \mathrm{C}$ for $1 \mathrm{~min}$, and $72^{\circ} \mathrm{C}$ for $3 \mathrm{~min}$. The PCR products after amplification were electrophoresed on $1 \%$ agarose gel and stained with ethidium bromide (Figure 2).

\section{Gene clean}

TIANgel Midi Purification Kit, Cat\# DP209-02.PCR was used according to manufacturer's instructions for elution of the PCR amplificated DNA.

\section{Sanger sequencing of the targeted mIDNA region}

DNA sequencing were carried out using the BigDye terminator cycle sequencing method and reaction products were analyzed on an ABI-Prism 377 automated sequencer at Macrogen Inc. Korea (www. macrogen.com).

The resulted sequencing data was analyzed using $\mathrm{NCBI}$ online tool BLAST. The revised Cambridge Reference Sequence (rCRS) of human mitochondrial DNA was used as reference for analysis of the DNA samples. 


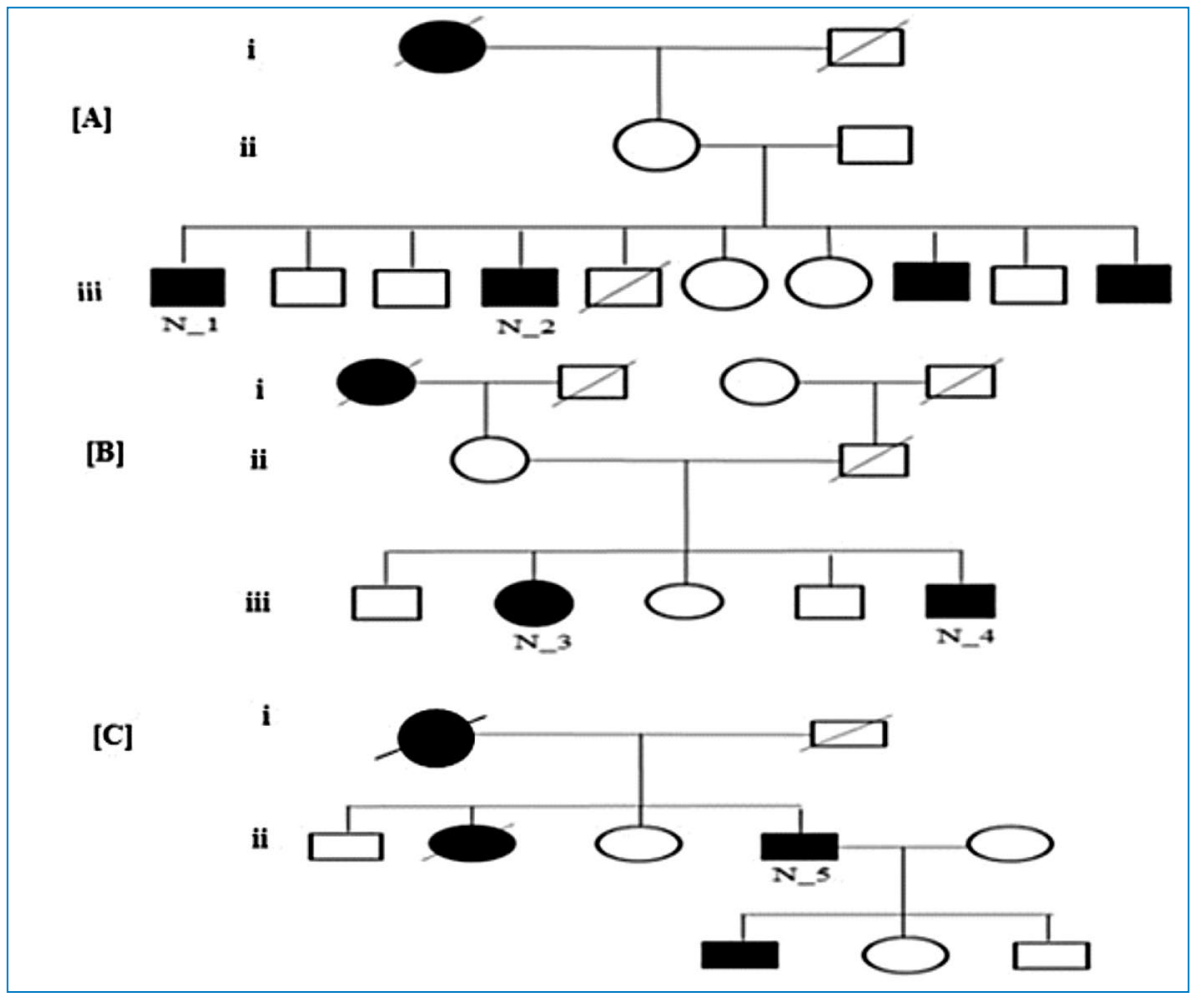

Figure 1. Family pedigree of all the three families (A, B and C) including three generations. The circles represent the female members while the squares representing the male members. Black square and circles represent the diseased one and white show the normal non diseased one. $\mathrm{N}_{-} 1$ to $\mathrm{N} \_5$ are the type 2 diabetes individuals inlcuded in the final analysis

Table 1. Characteristics and clinical history of all the study subjects $(\mathrm{N}=5)$

\begin{tabular}{|c|c|c|c|c|c|}
\hline Sample number & Gender & Age (years) & Weight $[\mathrm{kg}]$ & Complications & Medications \\
\hline N_1 & Male & 60 & 60 & Renal disease & Insulin \\
\hline N_2 & Male & 55 & 75 & $\begin{array}{l}\text { Blindness, joint problems, } \\
\text { high blood pressure }\end{array}$ & Oral hypoglycemic agent \\
\hline N_3 & Female & 50 & 65 & $\begin{array}{l}\text { Joint pain, infertility, } \\
\text { skin and renal disease }\end{array}$ & Insulin \\
\hline N_4 & Male & 43 & 80 & $\begin{array}{l}\text { High blood pressure, hearing loss, } \\
\text { renal and heart disease }\end{array}$ & Oral hypoglycemic agent \\
\hline N_5 & Male & 75 & 55 & High blood pressure, renal disease & Insulin \\
\hline
\end{tabular}

Table 2. DNA concentrations of samples $(\mathrm{N}=5)$

\begin{tabular}{lc}
\hline Sample number & DNA concentration $[\mathrm{ng} / \mu \mathrm{l}]$ \\
\hline N_1 & 1.81 \\
N_2 & 1.96 \\
N_3 & 1.78 \\
N_4 & 1.90 \\
N_5 & 1.84 \\
\hline
\end{tabular}

\section{Results}

Individual's clinical and social history was recorded with the help of questionnaires. Characteristics of the individuals and most common clinical complications related to type $2 \mathrm{DM}$ are presented in Table 1 . The tRNA ${ }^{\text {Leu(UUR) }}$ gene from the proband $N_{-} 1, N_{-} 2, N_{-} 4$ and N_5 from the diabetic families were sequenced. Sample 3 (N_3) was wasted and not included in the 


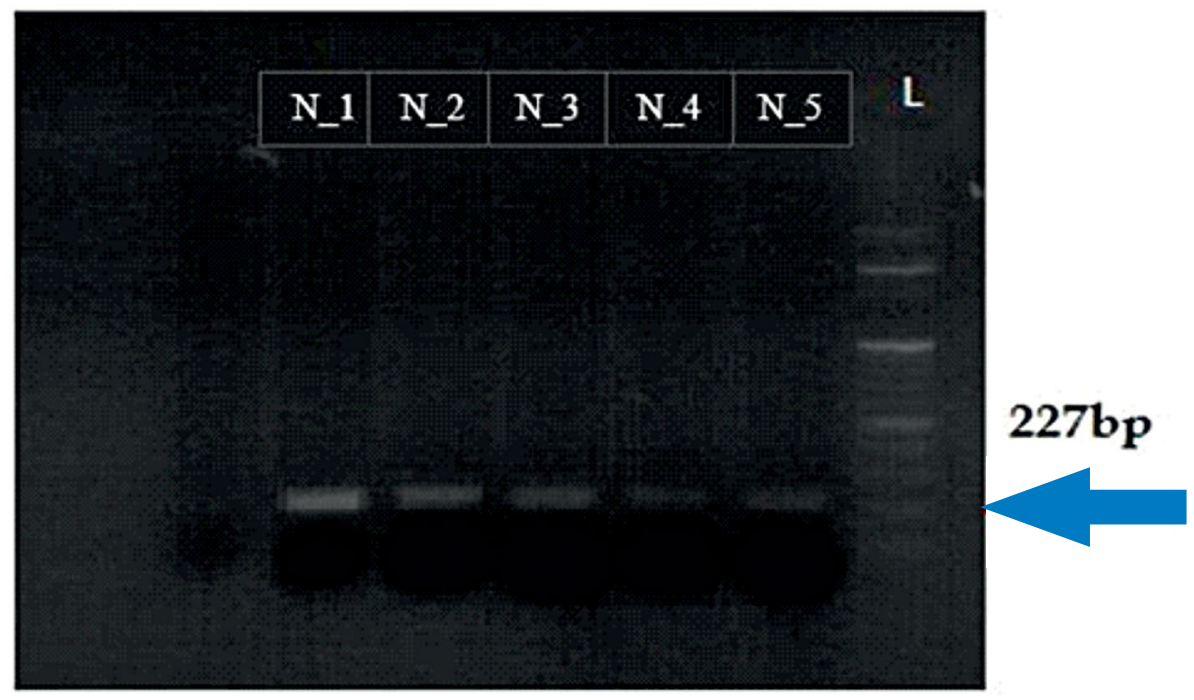

Figure 2. PCR amplified tRNA ${ }^{\text {Leu(UUR) }}$ gene products from sample $N_{-} 1$ to $N_{-} 5$ of 227 bp DNA fragments, $L=100$ bp DNA marker

final analysis. Sequences were aligned using online tool using NCBI. The obtained sequence data samples were aligned with rCRS with accession No NC-012920.1 (Figure 3). After careful analysis of sequenced data, point mutation tRNA ${ }^{\text {Leu(UUR) }}$ gene at position 3243 A-G was not observed.

\section{Discussions}

In Pakistan, presently 6.9 million individuals suffer from diabetes. It is assumed that by 2030, Pakistan will be ranked fifth for residing most number of DM individuals [14]. The inheritance of diabetes is different in different ethnic groups i.e. some ethnic groups in a particular area are more affected than others. Also, social, behavioral and environmental factors contribute to the onset of disease $[15,16]$. Pakistani tribal areas are still not explored with respect to mtDNA mutation associated with MIDD. In this research study, we assessed and analyzed mtDNA tRNA ${ }^{\text {Leu(UUR) }}$ gene mutation in families suffering from diabetes, living in tribal areas of Pakistan. During recording of the questionnaires, most of the individuals had experienced emotional stress before the onset of type 2 DM. Emotional stress is a risk factor for type $2 \mathrm{DM}$ that should also be considered [17]. The outcomes of the current study for mutation in mitochondrial tRNA ${ }^{\text {Leu(UUR) }}$ gene in subjects with type 2 DM in population from tribal areas of Pakistan identified none of the individual as a carrier of this mutation.

Diabetes mellitus is a group of metabolic disorders such as destruction of beta cells of pancreas and genomic DNA mutations in genes linked to type 2 DM [18]. Gene mutations are involved in peripheral insulin sensitivity and glucose induced insulin secretions.
mtDNA has 10 times more spontaneous mutations as compared with nuclear genome and is responsible for more than $80 \%$ of MELAS cases due to lack of protective histone and DNA repair system [19]. Therefore, mitochondrial DNA mutation may lead to impaired insulin secretion due to its role in glucose induced insulin secretion in pancreatic beta cells and pancreatic islets cells easily effected by disturbance of oxidative phosphorylation. Diabetes mellitus associated with mtDNA is transmitted maternally while the most common point mutation associated with DM was mitochondrial DNA tRNA gene (i.e. A3243G). Pakistani population is not explored in respect to disease associated with mitochondria particularly type $2 \mathrm{DM}$ in relation with $\mathrm{mt}$ tRNA-Leu gene [20]. It is still not explored that which type of mtDNA mutation is responsible for type $2 \mathrm{DM}$.

In mtDNA, point mutations can occur due to deletion, insertion and substitution of nucleotide so comprehensive analysis and screening of entire mtDNA is required. However, the ratio of mutated mtDNA varies between tissues in relation to wild type mtDNA, being high ratio in post mitotic tissues (pancreas, brain and skeletal muscles) while low in rapidly dividing tissues (blood leukocytes). According to a study from Hart et al., the defect in the mitochondrial tRNA ${ }^{\text {Leu(UUR) }}$ gene is associated with type 2 DM [11]. Martikainen et al. reported that $1 \%$ of $D M$ emergence associated with A3243G mutation in mtDNA tRNA ${ }^{\text {Leu(UUR) }}$ gene [21]. The response of impaired insulin to glucose in patients with tRNA ${ }^{\text {Leu(UUR) }}$ gene mutation is an early and critical abnormality in the development of type 2 DM [7]. However, it should not be excluded that other mutations such as tRNAGlu $14709 \mathrm{~T} \rightarrow \mathrm{C}$, ND-13316 G $\rightarrow$ A, 


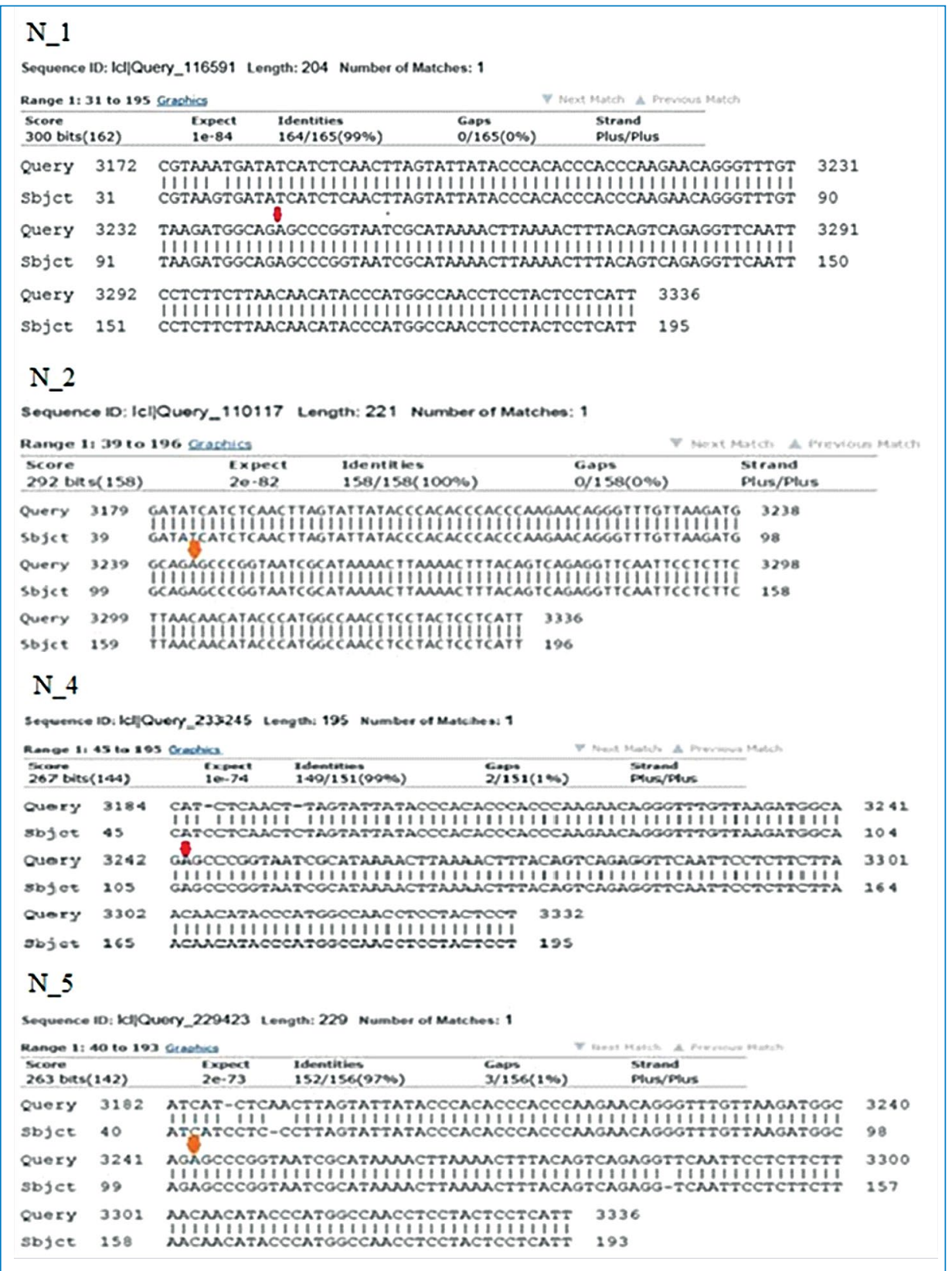

Figure 3. Sequence alignment of sample N_1 to N_5 obtained from Macrogen (www.macrogen.com) with revised Cambridge Reference Sequence (rCRS) of human mitochondrial DNA (Accession NO-012920.1)

$N D-13394 \mathrm{~T} \rightarrow \mathrm{C}, \mathrm{ND}-13426 \mathrm{~A} \rightarrow \mathrm{G}, \mathrm{ND}-412026 \mathrm{~A} \rightarrow \mathrm{G}$, tRNALeu $3256 \mathrm{C} \rightarrow \mathrm{T}$, tRNALys8296 $\mathrm{A} \rightarrow \mathrm{G}$, tRNALys8344 $A \rightarrow G$, tRNALys8363 G $\rightarrow$ A, tRNASer $12258 \mathrm{C} \rightarrow \mathrm{A}$ in genes have active role in etiology of disease [22].

The pathogenic mutation in the mitochondrial genome is very common due to the lack of efficient mitochondrial DNA mutation repair machinery. The mitochondrial genome has overlapping coding regions and mutation in any region can cause severe phenotypic effects. Since 1988, more than 270-point mutations have been described, affecting every mtDNA gene. Remarkably, more than half of these mutations are located in tRNA genes, even though tRNA comprise only about $10 \%$ of the total coding capacity of the genome. Among the point mutations the most common are an $A \rightarrow G$ transition at position 3243 in the tRNALeu(UUR) gene. It was long believed that $A \rightarrow G$ transition at position 3243 in the tRNA ${ }^{\text {Leu(UUR) }}$ gene is the common cause of type 2 DM $[23,24]$.

The leucine tRNA gene in the mitochondrial genome appears to be a frequent spot for point mutations, as several different mutations have been described so far. The most common mutation occurs at base pair (bp) 3243, and it accounts for approxi- 
mately $80 \%$ of cases of the MELAS syndrome. For this reason, mtDNA testing were preferred while MODY has a high inheritance rate and represents one end of a continuum of monogenic forms of diabetes that includes neonatal diabetes $[25,26]$. The frequency of the A3243G mutation in mitochondrial tRNA ${ }^{\text {Leu(UUR) }}$ gene vary in the members of different ethnicities. In a study conducted for the confirmation of A3243G mutation in tRNA ${ }^{\text {Leu(UUR) }}$ gene being the frequent cause of type $2 \mathrm{DM}$ has concluded that this mutation is not the frequent cause of type 2 DM [27].

In this study, all the selected families had type 2 DM familial vertical history. The clinical description included phenotypic features including sensorineural hearing loss, diabetes mellitus, cardiovascular disease, renal disease, blindness, arthritis, hypertension, and infertility. Other potential diabetic phenotypes include noninsulin-dependent diabetes mellitus (NIDDM), insulin-dependent diabetes mellitus (IDDM), malnutrition related diabetes mellitus (MRDM) and other diabetes associated syndromes such as Wolfram syndromediabetes insipidus, diabetes mellitus, optic atrophy, and deafness (DIDMOAD) and Maturity-onset diabetes of the young (MODY) [28]. NIDDM is a heterogeneous disorder with different pattern of inheritance that appears after forty year of age and is characterized by defect in beta cell function and insulin resistance [29]. IDDM is a polygenic disease characterized by defects in secretion and action of insulin. Several molecular alteration such as decrease in receptor tyrosine kinase activity, insulin receptor number, and IRS-1 phosphorylation contribute to insulin resistance [30]. MRDM also known as tropical pancreatic diabetes mellitus is rare diabetes linked with long term malnutrition, characterized by hyperglycaemia, insulin resistance, insulinopenia, and dysfunctional of the beta cells of pancreas [31]. DIDMOAD is a mitochondrial DNA disorder, can cause DM as the start of symptoms [32]. MODY is a monogenic disorders in seven different genes mutations lead to alter secretion of insulin [33]. Some diabetic patient, diagnosed as type 2 DM do not indicate evidence of circulating autoantibodies and overweight, are medicated using oral hypoglycemic drugs. This type of diabetes is classified as latent autoimmune diabetes of adults (LADA) [34].

The outcomes of the current study for mutation in mitochondrial tRNA ${ }^{\text {Leu(UUR) }}$ gene in subjects with type $2 \mathrm{DM}$ in population from tribal areas of Pakistan identified none of the individual as a carrier of A3243G mutation in tRNA ${ }^{\text {Leu(UUR) }}$ gene. The mitochondrial DNA is highly vulnerable to pathogenic mutation almost at any site, therefore in the etiology of this disease other gene variation should not be excluded. The clinical spectrum of mtDNA mutations are extremely broad, identical clinical signs and symptoms can be caused by nuclear genes and mtDNA mutations. If the suspect are obese or overweight with fasting hyperglycaemia, glycosuria in the presence of normoglycaemia and having strong family history of DM recommends mtDNA tests. Also, the large sample size and comprehensive sequencing of the entire mtDNA molecule is needed in Pakistani population.

\section{Acknowledgement}

Authors would like to thank the lab technician and volunteers who participated in this study.

\section{Conflict of interest}

The authors declare that they do not have any competing interests.

\section{REFERENCES}

1. Naveed A, Wahid M, Naveed A. Mitochondrial tRNALeu(UUR) gene mutation and maternally inherited diabetes mellitus in Pakistani population. Int J Diabetes Mellit. 2009; 1(1): 11-15, doi: 10.1016/j.ijdm.2009.03.012.

2. Greiner S, Sobanski J, Bock R. Why are most organelle genomes transmitted maternally? BioEssays. 2014; 37(1): 80-94, doi: 10.1002/bies.201400110

3. Wang M, Zhou XL, Liu RJ, et al. Multilevel functional and structural defects induced by two pathogenic mitochondrial tRNA mutations. Biochem J. 2013; 453(3): 455-465, doi: 10.1042/ bj20130294.

4. Li R, Guan MX. Human mitochondrial leucyl-trna synthetase corrects mitochondrial dysfunctions due to the tRNALeu(UUR) A3243G mutation, associated with mitochondrial encephalomyopathy, lactic acidosis, and stroke-like symptoms and diabetes. Mol Cell Biol. 2010; 30(9): 2147-2154, doi: 10.1128/mcb.01614-09.

5 . Guery B. The spectrum of systemic involvement in adults presenting with renal lesion and mitochondrial tRNA(Leu) gene mutation. Journal of the American Society of Nephrology. 2003; 14(8): 2099-2108, doi: 10.1097/01.asn.0000080180.51098.02.

6. Schon E, DiMauro S, Hirano M. Human mitochondrial DNA: roles of inherited and somatic mutations. Nat Rev Genet. 2012; 13(12): 878-890, doi: 10.1038/nrg3275.

7. Brandle $M$, Lehmann R, Maly FE, et al. Diminished insulin secretory response to glucose but normal insulin and glucagon secretory responses to arginine in a family with maternally inherited diabetes and deafness caused by mitochondrial tRNALEU(UUR) gene mutation. Diabetes Care. 2001; 24(7): 1253-1258, doi: 10.2337/ diacare.24.7.1253.

8. Gerbitz KD, Gempel K, Brdiczka D. Mitochondria and diabetes: genetic, biochemical, and clinical implications of the cellular energy circuit. Diabetes. 1996; 45(2): 113-126, doi: 10.2337/ diab.45.2.113.

9. Nile D, Brown A, Kumaheri M, et al. Age-Related mitochondrial DNA depletion and the impact on pancreatic beta cell function. PLoS ONE. 2014; 9(12): e115433, doi: 10.1371/journal. pone.0115433.

10. Schaefer A, Walker M, Turnbull D, et al. Endocrine disorders in mitochondrial disease. Mol Cell Endocrinol. 2013; 379(1-2): 2-11, doi: 10.1016/j.mce.2013.06.004.

11. Hart LM't, Hansen T, Rietveld I, et al. Evidence that the mitochondrial leucyl tRNA synthetase (LARS2) gene represents a novel type 2 diabetes susceptibility gene. Diabetes. 2005; 54(6): 1892-1895, doi: 10.2337/diabetes.54.6.1892. 
12. Park H, Davidson E, King M. The pathogenic A3243G mutation in human mitochondrial tRNALeu(UUR) decreases the efficiency of aminoacylationt. Biochemistry. 2003; 42(4): 958-964, doi: $10.1021 / \mathrm{bi026882r}$.

13. Aidar M, Line S. A simple and cost-effective protocol for DNA isolation from buccal epithelial cells. Braz Dent J. 2007; 18(2): 148-152, doi: 10.1590/s0103-64402007000200012.

14. Latif A, Ghafoor A, Wali A, et al. Did diabetes mellitus affect treatment outcome in drug-resistant tuberculosis patients in Pakistan from 2010 to 2014? Public Health Action. 2018; 8(1): 14-19, doi: 10.5588/pha.17.0098.

15. Reinehr T. Type 2 diabetes mellitus in children and adolescents. World J Diabetes. 2013; 4(6): 270-281, doi: 10.4239/wjd. v4.i6.270.

16. Razaq S, Khan MW, Masood Z, et al. et al.. An investigation on the prevalance of gestational diabetes mellitus in the pregnant women of province balochistan. WJMS. 2015; 12(2): 198-203, doi: 10.5829/idosi.wjms.2015.12.2.93229.

17. Brown LC, Majumdar SR, Newman SC, et al. History of depression increases risk of type 2 diabetes in younger adults. Diabetes Care. 2005; 28(5): 1063-1067, doi: 10.2337/diacare.28.5.1063.

18. Wahid M, Naveed AK, Hussain I. Insulin and glucagon ratio in the pathophysiology of diabetic ketoacidosis and hyperosmolar hyperglycemic non-ketotic diabetes. J Coll Physicians Surg Pak. 2006; 16(1): 11-14, doi: 1.2006/JCPSP.1114.

19. Lorenzoni $P$, Werneck $L$, Kay $C$, et al. When should MELAS (Mitochondrial myopathy, Encephalopathy, Lactic Acidosis, and Strokelike episodes) be the diagnosis? Arquivos de Neuro-Psiquiatria. 2015; 73(11): 959-967, doi: 10.1590/0004-282x20150154.

20. Jiang Z, Wu X, Zhu Y. Variant at position 10,055 in mitochondrial tRNAGly gene has a negative association with aplastic anemia. Mitochondrial DNA Part A. 2015; 27(5): 3086-3088, doi: 10.3109/19401736.2014.1003921.

21. Martikainen M, Rönnemaa T, Majamaa K. Prevalence of mitochondrial diabetes in southwestern Finland: a molecular epidemiological study. Acta Diabet. 2012; 50(5): 737-741, doi: 10.1007/ s00592-012-0393-2.

22. Wang $S$, Wu $S$, Zheng $T$, et al. Mitochondrial DNA mutations in diabetes mellitus patients in Chinese Han population. Gene. 2013; 531(2): 472-475, doi: 10.1016/j.gene.2013.09.019.

23. Abrar S, Muhammad K, Zaman $\mathrm{H}$, et al. Molecular genetic analysis of Type II diabetes associated m.3243A>G mitochondrial DNA mutation in a Pakistani family. Egyptian Journal of
Medical Human Genetics. 2017; 18(3): 305-308, doi: 10.1016/j. ejmhg.2016.12.001.

24. Zambelli A, Vidal-Rioja L. Lack of association between mitochondrial DNA mutation np3243 and maternally inherited diabetes mellitus. Clinical Biochemistry. 1999; 32(1): 81-82, doi: 10.1016/ s0009-9120(98)00102-7.

25. Rusanen H, Majamaa K, Tolonen U, et al. Demyelinating polyneuropathy in a patient with the tRNALeu(uur) mutation at base pair 3243 of the mitochondrial DNA. Neurology. 1995; 45(6): 1188-1192, doi: 10.1212/wnl.45.6.1188.

26. Mohan V, Radha V, Nguyen T, et al. Comprehensive genomic analysis identifies pathogenic variants in maturity-onset diabetes of the young (MODY) patients in South India. BMC Med Genet. 2018; 19(1): 22, doi: 10.1186/s12881-018-0528-6.

27. Malecki M, Klupa T, Wanic K, et al. Search for mitochondrial A3243G tRNALeu mutation in Polish patients with type 2 diabetes mellitus. Med Sci Monit. 2001; 7(2): 246-250; PMID: 421154.

28. Kuzuya T, Matsuda A. Classification of diabetes on the basis of etiologies versus degree of insulin deficiency. Diabetes Care. 1997; 20(2): 219-220, doi: 10.2337/diacare.20.2.219.

29. Lehto M, Tuomi T, Mahtani MM, et al. Characterization of the MODY3 phenotype. Early-onset diabetes caused by an insulin secretion defect. J Clin Invest. 1997; 99(4): 582-591, doi: 10.1172/ jci119199.

30. Guerra C, Navarro P, Valverde A, et al. Brown adipose tissue-specific insulin receptor knockout shows diabetic phenotype without insulin resistance. J Clin Invest. 2001; 108(8): 1205-1213, doi: 10.1172/jci13103.

31. Bhirud P. BalasahebBJ. Conceptual study of malnutrition related diabetes mellitus. JAIMS. 2019; 4(1): 89-95, doi: https://doi. org/10.21760/jaims.4.1.18.

32. Rötig A, Cormier V, Chatelain P, et al. Deletion of mitochondrial DNA in a case of early-onset diabetes mellitus, optic atrophy, and deafness (Wolfram syndrome, MIM 222300). J Clin Invest. 1993; 91(3): 1095-1098, doi: 10.1172/jci116267.

33. Schober E, Rami B, Grabert M, et al. Phenotypical aspects of maturity-onset diabetes of the young (MODY diabetes) in comparison with Type 2 diabetes mellitus (T2DM) in children and adolescents: experience from a large multicentre database. Diabet Med. 2009; 26(5): 466-473, doi: 10.1111/j.1464-5491.2009.02720.x.

34. Catchpole B, Kennedy $\amalg$, Davison $\sqcup$, et al. Canine diabetes mellitus: from phenotype to genotype. J Small Anim Pract. 2008; 49(1): 4-10, doi: 10.1111/j.1748-5827.2007.00398.x. 